\title{
Extended Semantic Annotations for Generating Translators in the Arrowhead Framework
}

\author{
Filipe Moutinho, Luís Paiva, Julius Köpke, Pedro Maló
}

\begin{abstract}
In order to create distributed automation systems, it is required to ensure their interoperability; however, ensuring interoperability between heterogeneous systems (using different communication protocols, data formats, and semantics) is a challenging task. Among many interoperability challenges, this paper addresses issues concerning semantic and data interoperability, namely, it provides a contribution to support the semantic compatibility verification and the generation of translators for XML messages. Translators are generated based on XML-Schemas that are annotated with a reference ontology. We base our annotations on an extension of an existing declarative annotation method. In particular our extension explicitly addresses ambiguities of annotations, schema instance mismatches and coverage mismatches that frequently occur on IoT message schemas. We have evaluated our approach based on a set of interaction scenarios from the domain of the Arrowhead Project. A tool prototype that supports the semantic compatibility verification and the generation of XML translators is available at http://gres.uninova.pt/tag/.
\end{abstract}

Index Terms-Cyber-Physical Systems, Internet-of-Things, Interoperability, Message Transformation, Translators Automatic Generation, XML-Schemas, Semantic Annotations, Semantic \& Ontology Reasoning, Knowledge-Based Systems.

\section{INTRODUCTION AND MOTIVATION}

D ISTRIBUTED automation systems [1], Cyber-Physical Systems (CPS) [2], and Internet-of-Things (IoT) [3], [4], commonly have several physical devices/sensors and systems connected to each other. This means that there is a need to interoperate seamlessly with all actors, i.e., hardware, software or humans from within an environment or even outside. As such, for two actors to interoperate they need to have a shared understanding of the data exchanged. Achieving a shared understanding is a non trivial task since a myriad of technologies and protocols, aimed at device communication, exist. For instance, in a real-world system, temperature sensors need to measure temperature and send their readings, e.g., a numeric value and its units to another device waiting for them. IoT and CPS frameworks try to harmonize both worlds,

F. Moutinho and P. Maló (e-mail: fcm@fct.unl.pt, pmm@fct.unl.pt) are with Dept. of Electrical Engineering, Faculty of Science and Technology, Nova University of Lisbon, 2829-516 Caparica, Portugal.

F. Moutinho, L. Paiva (e-mail: luismpaiva@uninova.pt), and P. Maló are with Center of Technology and Systems, UNINOVA, Faculty of Science and Technology, Nova University of Lisbon, 2829-516 Caparica, Portugal.

J. Köpke (e-mail: julius.koepke@aau.at) is with the Department of Informatics-Systems, University of Klagenfurt, Austria.

This work was partially financed by Portuguese Agency "Fundação para a Ciência e a Tecnologia" (FCT) and by EU ARTEMIS and ECSEL JU fundings, under grant agreements nr. 332987 (Arrowhead project), nr. 662189 (MANTIS project), and nr. 737459 (Productive4.0 project), and by Portuguese Agency FCT, in the framework of project UID/EEA/00066/2013.

Manuscript received May 01, 2017; revised September 11, 2017; accepted November 12, 2017. proposing solutions for solving these issues. One of IoT and CPS requirements is the ability to connect heterogeneous ecosystems [2][5]. In other words, the capacity they should have to interoperate with each other on demand and as needed. Specifically, this capacity is referred to as Dynamic Interoperability [6]. Dynamic Interoperability deals with the sustainability of interoperability in diverse CPS working in dynamic, large scale environments.

EU promoted a CPS related initiative called Arrowhead Project [7][8], based on a vision of collaborative automation by networked embedded devices. The Arrowhead Framework [7][8] is a Service-Oriented Architecture (SOA) framework that enables interaction between several systems. For a consumer to request and receive a service, it needs to discover a provider, who offers the desired services, as well as needs to be authorized to communicate with it. It is important to note that in this paper, when we refer to devices/systems, we mainly use the word systems.

The Arrowhead Framework (AF) provides several core services, such as service registry (SR) and discovery (SD), orchestration (ORC), as well as authorization (AUT), to be used by devices/systems. The usual interaction between devices/systems and the AF core services, is as follows: (1) SR is responsible to receive all registry requests from systems (i.e., providers), having services available to offer; (2) Systems which need to consume services (i.e., consumers), use SD to search for providers with the services required; (3) ORC service delivers information about the best providers for the services requested by consumers; (4) Responsible for providing authorization information, is AUT service, which is aimed at the authorization process for the communication between consumers and providers. The typical interaction between a provider, a consumer and an AF is illustrated in Fig. 1.

Any kind of system that wants to use the Arrowhead Framework must comply to its specifications. In other words, new systems can be built specifically customized to the framework specifications, resulting in full (or total) compliance. However, legacy systems need to adapt, by changing their own specifications or by using an adapter. Such an adapter is a sufficient condition to support interaction between Arrowhead Framework core services and embedded systems. On the other hand, to support interaction between systems, (e.g., provider/consumer interactions) a single adapter is not enough. In order to fully support interaction between systems (for example, between a provider and several heterogeneous consumers), the need to use several converters/translators arises. Such necessity results from a myriad of communication protocols a device can have (e.g., HTTP, MQTT, XMPP, COAP, ...), data formats 


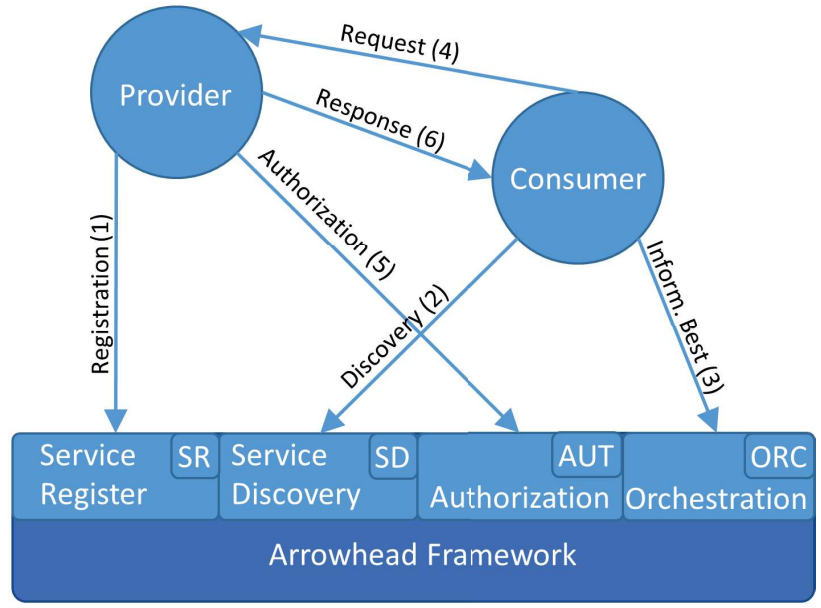

Fig. 1. Interaction between a provider, a consumer and an Arrowhead Framework

(e.g., XML, CSV, JSON, ...), as well as semantics. These represent three interoperability dimensions, namely technical (protocols), syntactic (data formats) and semantic interoperability [6]. "Semantic interoperability means that there is a shared understanding between communicating 'things' on the meaning of the content (information) being exchanged" [9]. This paper focuses on the Semantic Interoperability Challenges, although Arrowhead Project deals with all three.

Translators are required, whenever a pair of systems that want to interact, send/understand XML messages that: (1) have different structures; (2) use different keywords to represent the same concept; and/or (3) do not share the same understanding of similar concepts, i.e., different semantics. Such translator may also add missing data required by consumers.

A specific translator (Fig. 2) is required for each pair of systems that want to communicate but use heterogeneous XML messages. Additionally, it is easy to realize that, if a large Number of Systems (NS) with heterogeneous XML messages want to interact, the possible number of systems pairs is even larger. This means that it would be a huge task to manually create each specific translator for each pair of systems. For instance, if there is a set of 100 systems (with heterogeneous XML messages), where each one wants to send and receive messages to/from the other 99 systems, the Number of different Translators $(N T)$, required to support their interaction, is a permutation of 100 in pairs: $N T=$ $N S^{2}-N S=100^{2}-100=9900$ translators. The problem of creating a large number of translators can be solved if they are automatically generated, as proposed in this paper.

\section{Example}

The following running example presents a scenario where one provider sends XML messages with data about two outdoor temperature sensors and one consumer is expecting to receive XML messages with data about one or more temperature sensors. The provider sends, for each outdoor sensor, the temperature value and units, as listed in Listing 1 ; whereas, the consumer, for each sensor, must receive the temperature value and units and the sensor location, as listed in Listing 2. The provider sensors are fixed (always at the

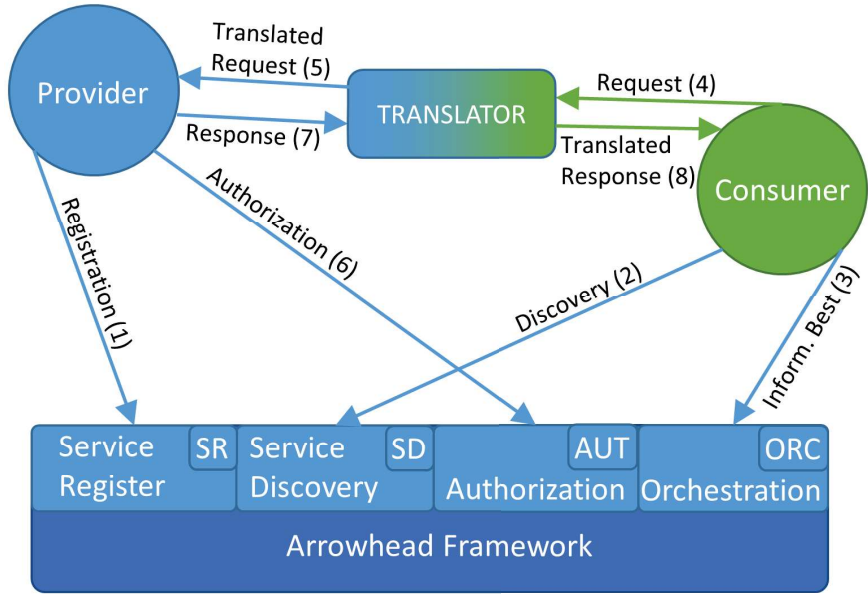

Fig. 2. Interaction between a provider, a consumer and an Arrowhead Framework, using a translator

same location).

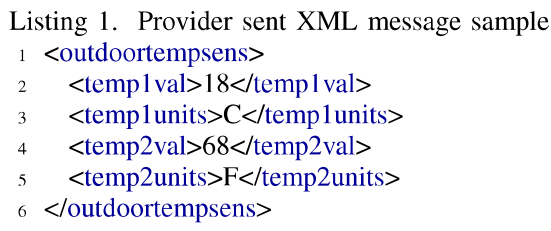

Listing 2. Consumer expected XML message sample $1<$ temperaturesensors>

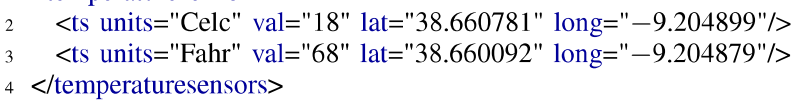

The translation of XML elements/attributes with different names, semantics, and data values, can be supported by existing annotation methods; however, the translation of XML messages where different XML elements/attributes need to be linked together or where complement data is required, was not supported by the existing annotation methods. The translation of the provider "outdoor temperature values" into consumer "temperature values" is supported by path based semantic annotations, proposed in [10], [11]; whereas the mapping of the provider temperature units ("C" and "F") to the consumer temperature units ("Celc" and "Fahr"), is supported by semantically annotated data values, proposed in [12]. This paper proposes to support the association of each temperature value with its units ("temp1val" with "temp1units" and "temp2val" with "temp2units"), and a way to add the provider sensors location to the XSD, to be used by the translator to create XML messages for the consumer.

\section{Contributions}

This paper proposes: (1) the use of XML-Schemas (XSDs) with semantic annotations and data values; (2) a method to group semantic annotations, creating data associations; (3) a method to add complement data values. A tool that receives as inputs two XSDs and a reference ontology in OWL (Web Ontology Language) [13] format, performing the semantic compatibility verification and, if possible, the generation of XML translators, have been developed during this work. This tool prototype is available online at http://gres.uninova.pt/tag/. 
The present document is structured as follows. The related work, where the semantic annotation of XML-Schemas is discussed, is presented in section II. Section III is composed of four subsections, where the first two present XSD annotations proposed in other papers ([10], [11], [12]) and the other two subsections present the enhanced annotation method proposed in this paper. The developed tool is briefly described in section IV, whereas an experimental evaluation is presented in section V. Finally, conclusions are presented in section VI.

\section{RELATED WORK}

In general, the generation of translators between different message formats (schemas) requires knowledge about the semantics of schema elements. One way to provide explicit semantics is to annotate schemas with a reference ontology. This approach is applied in the Arrowhead Framework and has the advantage that sensors sending data and consumers requesting data do not need to be adopted in order to participate. Instead, only the corresponding XML-Schemas need to be annotated. While semantic annotations at the instance level such as [14], [15], [16] and standards such as RDFa [17], Micro Formats $^{1}$ and Open Annotation [18] received a lot of attention, annotations of XML-Schemas were addressed in a much lesser degree. The main standard for semantic annotations of XSD is SAWSDL [19] of the W3C. SAWSDL is an embedded semantic annotation method for XML-Schema and WSDL. SAWSDL annotations come in two flavors: model references and lifting/lowering mappings. Lifting and lowering mappings are references to scripts that transform instance documents of annotated schemas to and from instance documents of some semantic model. Such scripts may be formulated in any language (e.g., XSLT, XQuery, ...). Transformations based on this approach first transform every instance document to an instance of the semantic model and then transform it back to an instance of the target schema. While this method is very expressive it has disadvantages in terms of scalability, maintainability and also in terms of ease of use for annotators. In contrast, model references are attached to XML Schema types, attributes and elements and establish references to concepts of an ontology. Lifting and lowering mappings are intended for runtime transformations and model references are intended for service discovery in [19]. However, such lightweight annotations in form of model references are easily applicable and also allow document transformations using a typical matching and mapping workflow in the spirit of the Clio [20] system. Therefore, in the matching step semantically similar elements are matched based on the annotations employing ontological reasoning and the match result can then be used for mapping systems [21] that actually derive executable mappings employing the theory of data exchange [22].

Unfortunately, pure model references (as proposed in SAWSDL) can only point to named concepts of a reference ontology. This has disadvantages in terms of expressivity when general reference ontologies are used for annotations. Expressive and therefore precise annotations are only possible if very specific reference ontologies providing named concepts for all

${ }^{1}$ http://microformats.org schema nodes are provided. Annotation paths [10], [23], [24], [11] were developed to overcome this limitation. Annotation paths are path expressions over concepts and properties of DL (Description Logic) reference ontologies (e.g., OWL DL ontologies [13]). Such path expressions translate to complex DL concepts precisely defining the meaning of the schema nodes. Matching systems operating on annotation paths [11], [25] are based on the so called extended reference ontology containing all OWL ontology concepts defined in the annotation paths. A recent evaluation of annotation paths in [11] has shown superior match quality compared to matching based on pure model references and also compared to traditional schema matching approaches such as [26], [27], [28], [29], [30], [31].

However, annotation paths were developed primarily for business documents and assume that schemas typically do not contain semantically identical information twice. Therefore, handling ambiguous annotations is not supported on the annotation level. Instead, existing matching systems operating on annotation paths [11], [25] apply heuristics based on the structure of the XML-Schemas or of the annotations to resolve ambiguities. Another problem are schema instance or subclass attribute mismatches [32]. In this case, instance documents actually encode schema information. E.g., in the example document in Listing 1, the value $C$ in the field templunits defines that the field temp1val contains a temperature value in Celsius. Finally, coverage mismatches where some data is missing in the source schema cannot be solved solely using annotations. Such mismatches are resolved by userdefined transformation templates in current annotation path based matchers [11], [25].

In the domain of the Arrowhead Framework, these problems frequently occur in messages exchanged between producers and consumers, demanding for an explicit annotation-level solution. Therefore, an extension of annotation paths to annotate potential instance data values on the schema level was proposed in [12]. In this paper, we extend the work of [12], in order to have: (1) annotation paths to deal with ambiguous annotations on the annotation level using group identifiers and (2) an annotation method to aid the resolution of coverage mismatches, by explicitly adding missing data to schemas.

Other declarative annotation methods for schemas were proposed in [33] and [34]. The work in [33] which is based on a general work on annotations and mismatches in [32] targets the annotation of RDFS Schemas. The approach uses complex DL expressions for formal annotations which have a similar structure as the DL representation of annotation path expressions. However, they follow a different aim for data transformations. Instead of directly generating translators, annotations are used to generate lifting and lowering mappings. A case study of the approach can be found in [35].

An external annotation method for DTDs is presented in [34]. In the approach annotations are graphs composed of elements of the DTDs labelled with concepts or data-type properties of an ontology. Edges are labelled with object-properties. Some rules for deriving simple matches are provided. The approach does not deal with complex matches as addressed by annotation paths in [11], [25] and does not provide means to define semantics of instance data values or to address coverage 
mismatches as proposed in this paper.

\section{XSDS WITH GROUPED SEMANTIC ANNOTATIONS AND DATA VALUES}

To support the semantic and data compatibility verification and the generation of XML translators, XSDs with the annotations described in this section, should be used. The annotations described in the subsections $C$ and $D$ are new (proposed in this paper). This section is composed of four subsections:

- A. Path Based Semantic Annotations - describes the semantic annotation method proposed in [10], [11];

- B. Mapping data values to ontology individuals - describes a method proposed in [12] to provide meaning to instance data values;

- C. Grouping semantic annotations in XSDs - proposes the introduction of group identifiers (IDs) in the Path Based Semantic Annotations to avoid ambiguities; and

- D. Complement data in XSDs - proposes an extension that allows to annotate schemas with static instance data values that may be required by consumers.

\section{A. Path Based Semantic Annotations}

Standard SAWSDL model references can only point to named concepts of an ontology. As a result the direct annotation of XML-Schemas with general reference ontologies does not allow for precise annotations [10], [11]. Therefore, the annotation paths method was developed in [10], [11]. Annotation Paths are paths through the reference ontology that are composed of concepts and properties. The string representation of annotation paths is directly used as SAWSDL model references. Such references point to the virtually extended reference ontology containing the equivalent $D L$ representations. We shortly describe the essence of the approach here and refer the reader to [10][11] for all details.

Example: Using the reference ontology in Fig. 3, a standard SAWSDL Model Reference can only refer to existing named concepts and not to properties. The XML attribute lat in Listing 2 can only be annotated with the named concept LATITUDE. Unfortunately, this does not express, that attribute values actually represent the latitude of some temperature sensor. A matcher may only heuristically infer that the latitude might be associated to some temperature element by inspecting the schema structure. However, this is not always possible (e.g., assume a completely flat schema). Using the annotation paths method the attribute latitude can be annotated with the annotation path TemperatureSensor/hasGPSLocation/LATITUDE. This annotation specifies a $D L$ subconcept of the LATITUDE concept: LATITUDE $\square \exists$ hasGPSLocation ${ }^{-}$.TemperatureSensor. Therefore, a LATITUDE concept that has some inverse hasGPSLocation property to some TemperatureSensor. To be even more precise, a longer path can be used that defines that the field actually carries a value property of a LATITUDE concept: /TemperatureSensor/hasGPSLocation/LATITUDE/hasValue. Steps of annotation paths referring to concepts may have additional restrictions. Such restrictions can restrict the concept to an enumerated class of individuals or they can impose existential restrictions on properties which are again formulated in form of path expressions.

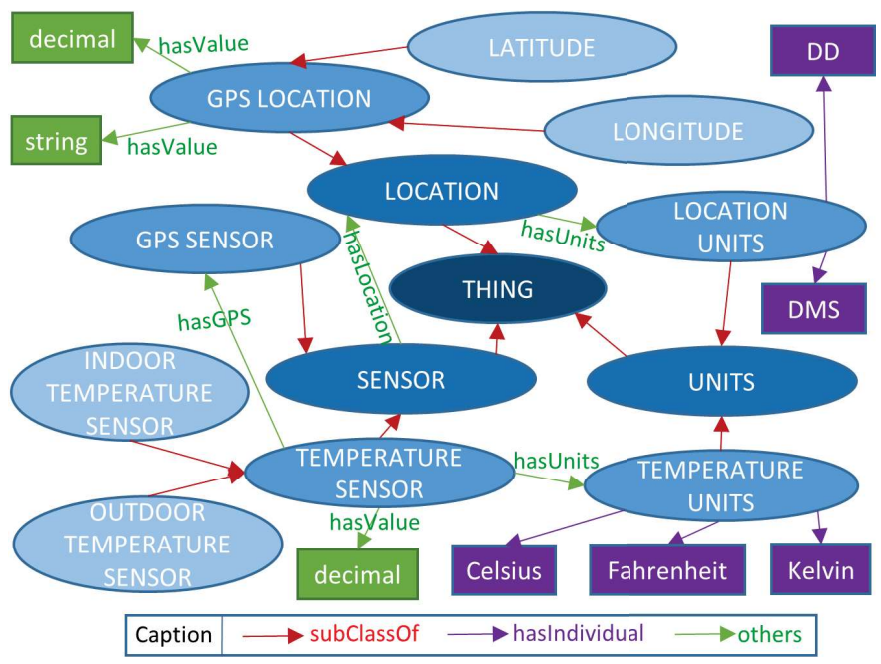

Fig. 3. Sample ontology of temperature sensors

1) Formal Definition of Annotation Paths: We repeat the essence of the annotation path method from [11] here. In order to define the annotation method, we first introduce a definition of the reference ontology. Annotation paths are tailored to be used together with description logics ontologies e.g., OWL DL ontology [13]. We provide a definition of DL ontologies that only covers the relevant aspects for the definition of annotation paths in this paper and refer the reader to [13], [36] for details.

Definition 3.1: Ontology [11]: An ontology $O$ is a tuple $O=(C, D P, O P, I, A)$, where $C$ is a set of concepts, $D P$ is a set of datatype-properties, $O P$ a set of object-properties, $I$ a set of individuals and $A$ a set of axioms over $C, D P, O P$, and $I$. Each element in $C, D P, O P$, and I is identified by a string that represents the URI of the specific element. The sets $C, D P, I$, and $O P$ are disjoint.

We have already shown the string representation of annotation paths in the example above. The string representation is just a sequence of steps delimited by a slash. Each step refers to a concept or property from the reference ontology. In general, an annotation path is defined as:

Definition 3.2: Annotation Path [11]: An annotation path $p$ for some reference ontology $O$ is a sequence of steps $p=\left(s_{1}, s_{2}, \ldots, s_{n}\right)$. Each step $s$ in $p$ is a tuple of the form (uri, type, res), where s.uri is an URI defined in the reference ontology: s.uri $\in O . C \cup O . O P \cup O . D P$. The type of a step s.type can be cs (concept-step), op (object-property) step or $d p$ (datatype-property-step). It is defined by the type of the referenced element in O: s.uri $\in$ O.C $\Rightarrow$ s.type $=c s$; s.uri $\in O . O P \Rightarrow$ s.type $=$ op; s.uri $\in O . D P \Rightarrow$ s.type $=d p$.

The function length maps annotation paths to their number of steps. The type function $t$ maps annotation paths to their types. Types are determined by the last step: 
$t(p)= \begin{cases}c a & \text { if } p(\text { length }(p)) \cdot \text { type }=c s \\ d p a & \text { if } p(\text { length }(p)) \cdot \text { type }=d p\end{cases}$

A restriction of a concept step $s$, s.res is a set of restriction elements. A restriction element is a tuple (type, value), where type $\in\{$ indv,path $\}$; type $=$ indv indicates that value contains a set of URIs pointing to ontology individuals, while type $=$ path indicates that value contains an annotation path. In the string representation, we denote sets of individuals in curly brackets. Restriction paths are written as they are. Multiple restriction elements are delimited by colon.

Definition 3.3: An annotation path $p$ is structurally valid if all of the following conditions hold [11]:

- $p(1) . t y p e=c s$ - The first step must refer to a concept.

- $p($ length $(p))$.type $\in\{d p, c s\}$ - The last step must refer to a concept or a datatype-property.

- $\forall i \in\{1 .$. length $(p)-1\}: p(i)$. type $=c s \Rightarrow p(i+$ 1).type $\in\{d p, o p\}$ - The successor of a concept-step must be an object-property or datatype-property-step.

- $\forall i \in\{2 .$. length $(p)-1\}: p(i)$.type $=o p \Rightarrow p(i+$ 1).type $\in\{c s\}$ - The successor of an object-propertystep must be a concept-step.

- $\forall i \in\{1$..length $(p)\}: p(i)$. type $=d p \Rightarrow$ length $(p)=i$ - Only the last step can refer to a datatype-property.

- $\forall i \in\{1$..length $(p)\}: p(i)$.res $\neq\{\} \Rightarrow p(i)$.type $=c s$ Only concept steps may have restrictions.

- $\forall i \in\{1$..length $(p)\}: p(i)$.res $\neq\{\} \Rightarrow \operatorname{valid}(p(i)$.res $)$ - All restrictions must be structurally valid (see Def. 3.4).

Definition 3.4: A restriction of a concept step $s$ is structurally valid if [11]:

$\forall r \in$ s.res :

- $r . t y p e=i n d v \Rightarrow \forall i \in$ r.value $\Rightarrow i \in O . I$

- r.type $=$ path $\Rightarrow$ structvalid(r.value) (see Def. 3.3)

An annotation path is logically valid, if the representation of the path as a $D L$ concept does not contradict with the ontology. Therefore, the corresponding concept is satisfiable in the ontology. We refer the reader to [23], [11] for all details.

\section{B. Mapping data values to ontology individuals}

Path based semantic annotations provide meaning to XSD elements; however, they do not provide meaning to data values of those XSD elements in the XML instance files. For example, the XML element "templunits" from Listing 1, can be annotated, in the associated XSD, with the annotation path /OutdoorTemperatureSensor/hasUnits/TemperatureUnits, giving meaning to the XML element but not to the values that this XML element can contain. This XML element can contain two different values for temperature units: " $C$ " and " $F$ ", but to support the translation, their meaning, which is Celsius and Fahrenheit, must be specified, for instance, in the XSD. An annotation method, to semantically annotate XML data values in the XSDs, was proposed in [12].

The annotation method proposed in [12] provides a way to map XML data values to ontology individuals, providing meaning to those values. The required mappings for this paper running example are presented in the XSD annotations (xs:annotation/xs:appinfo) of Listing 3 and Listing 4. Each mapping (a3st:map-data-ind) has an identifier (a3st:mdi-id), an ontology individual (a3st:individual), and an XML data value, which is the value of the a3st:map-data-ind element.

It is also required to identify for each XSD element which mappings apply. To do it, it is required to add the attribute a3st:mdi-ref into the XSD element with a list of the mapping identifiers. If the provider and the consumer temperature units are mapped into the ontology individuals, and given that they both send/receive temperature values in Celsius or Fahrenheit, it is possible to translate the provider units values $(C$ and $F$ ) to the consumer units values (Celc and Fahr).

\section{Grouping semantic annotations in XSDs}

Using path based semantic annotations [10], [11], it is possible to provide meaning to each XML element and attribute, through semantic annotations in the XSDs; however, it is not possible to group/associate XML elements/attributes. For instance, the provider that sends the XML message presented in Listing 1, sends two temperature values and two temperature units, but just using path based semantic annotations, it is not possible to associate the element tempIval with the element templunits nor the temp2val with the temp2units. Assuming that both sensors have the same semantics, it must be ensured that the translator does not mix their data and, it is not reliable for a translator to make such association without additional information about the provider message.

To avoid ambiguities and enable the automatic generation of translators, the introduction of Group Identifiers (IDs) in the XSD semantic annotations, is proposed and defined in this section. These group IDs should be used if:

- the schema contains elements with equivalent semantics (with regard to the reference ontology) and does not provide any structuring (like parent elements for sensor values); or

- the schema contains elements with equivalent semantics and one wants to apply a matching/mapping system that only operates on semantic annotations, not relying on structural information of the schemas.

The definitions proposed in this subsection, to include group IDs in path based semantic annotations, extend the definitions presented in subsection III-A.

Definition 3.5: Annotation Path with Groups: This definition extends Definition 3.2 with group IDs. In an annotation path with groups, each step $s$ in $p$ can also have a set of groups $s=\left(\right.$ uri,type, res, $\left.G^{\prime}\right)$, where $G^{\prime}$ is a subset of all "Concept Group Identifiers" $(C G I): G^{\prime} \subseteq C G I$.

Definition 3.6: An annotation path with groups $p$ is structurally valid if: all the conditions from Definition 3.3 are true and the following additional condition is satisfied:

- only concepts steps can have group ID $\left(\forall s \in p \mid\right.$ s.type $\left.\neq c s \rightarrow s . G^{\prime}=\emptyset\right)$.

Group IDs are only valid for one schema. E.g., matching groups between schemas is based on groups with similar members and not on equivalent group IDs (group IDs in provider and consumer schemas are independent). 
Only schemas with ambiguous information require group IDs. For instance, if an XML message contains data about sensors, the group IDs can be omitted when: (1) all data is about the same sensor; or (2) there is a repeated XSD element ("maxOccurs" > 1, such as in Listing 3) and all data, about one sensor, is in its sub-elements and attributes.

The group IDs are inserted in the annotation path string between braces, after the concept name, as illustrated in the XSDs, of the running example, presented in listings 3 and 4. When a single concept belongs to more than one group, their IDs must be separated by commas.

\section{Complement data in XSDs}

When a consumer requires more data than the one that is sent by the provider, the systems cannot interoperate, except if an intermediary adds that data. This intermediary should be the translator. The running example presents a scenario where the consumer requires the temperature sensor locations (latitude and longitude) but the provider does not provide them. If the provider sensor locations is always the same, their location can be inserted in the XML-Schemas and used to automatically generate the translator. Then the translator can create the translated XML messages with that missing data, which are data property values.

The namespace Annotating Schemas to Support Semantic Translations (A3ST), defined by the URI http://gres.uninova.pt/a3st, was extended during this work, to enable the introduction of data property values in XSDs. The following elements and attributes were added into the A3ST:

- data-property-value - this element, which must be a child of the "xs:appinfo", is used to add datatype property values about XML messages into their XSDs;

- property - an attribute of the element data-propertyvalue. Its value is a path based semantic annotation where the last step is a datatype property step;

- value - another attribute of the element data-propertyvalue. It is the value of the datatype property.

The provider XSD with the temperature sensor locations (latitudes and longitudes), as XSD annotations (xs:annotation), is presented in Listing 3.

\footnotetext{
Listing 3. The provider XSD with the grouped semantic annotations and data values

$11<$ <3st:data-property-value a3st:property="/TemperatureSensor $\{1\}$ / hasLocation/Latitude[hasUnits/LocationUnits/hasIndividual/DD]/ has Value" a3st:value="38.660781"/>

$12<$ a3st:data-property-value a3st:property="/TemperatureSensor $\{1\} /$ hasLocation/Longitude[hasUnits/LocationUnits/hasIndividual/ DD]/hasValue" a3st:value="-9.204899"/>

$13<$ <3st:data-property-value a3st:property="/TemperatureSensor $\{2\} /$ hasLocation/Latitude[hasUnits/LocationUnits/hasIndividual/DD]/ has Value" a3st:value="38.660092"/>

$14<$ a3st:data - property - value a3st:property="/TemperatureSensor $\{2\} /$ hasLocation/Longitude[hasUnits/LocationUnits/hasIndividual/ DD]/hasValue" a3st:value $="-9.204879 " />$

$15<$ xs:appinfo $></$ xs:annotation $>$

$16</$ xs:schema $>$

Listing 4. The consumer XSD with the grouped semantic annotations and data values

1 <xs:schema xmlns:xs="http://www.w3.org/2001/XMLSchema">

$<$ xs:element name $=$ "sensors" $><x s:$ complexType $><x s$ :sequence $>$ $<x s$ :element name $="$ temperature" minOccurs $=" 1 "$ maxOccurs $="$ unbounded">

$<$ xs:complexType>

$<x s:$ attribute type="xs:string" name="units" sawsdl modelReference $="$ /TemperatureSensor/hasUnits/ TemperatureUnits"/>

$<x s$ :attribute type="xs:decimal" name="value" sawsdl: modelReference="/TemperatureSensor/hasValue"/> $<$ xs:attribute type="xs:decimal" name="latitude" sawsdl: modelReference="/TemperatureSensor/hasLocation/Latitude/ has Value[/LocationUnits/hasIndividual/DD]"/>

$<x$ s:attribute type="xs:decimal" name="longitude" sawsdl: modelReference="/TemperatureSensor/hasLocation/ Longitude/hasValue[/LocationUnits/hasIndividual/DD]"/> $</$ xs:complexType $>$

$<$ xs:element $>$

$</$ xs:sequence $></$ xs:complexType $></$ xs:element $>$

$<$ xs:annotation $><x$ s:appinfo $>$

<a3st:map-data-ind a3st:mdi-id="1" a3st:individual="Fahrenheit"> Fahr $</$ a3st:map - data-ind $>$

14 <a3st:map - data-ind a3st:mdi-id="2" a3st:individual="Celsius"> Celc $</$ a3st:map-data-ind $>$

$15</$ xs:appinfo $><$ xs:annotation $>$

$16</$ xs:schema $>$

\section{TOOL PROTOTYPE}

As a proof of concept of the ideas proposed herein, a software tool prototype was developed. This tool supports: (1) the semantic and data compatibility verification and (2) the generation XML translators, namely it generates XSLTs (eXtensible Stylesheet Language Transformations) [37] that translate the provider XML messages to the consumers XML messages. The developed tool executes the following steps:

1) receives as inputs a pair of XSD files (one for a provider and another for a consumer) and an OWL (Web Ontology Language) [13] file for a domain ontology;

2) parses the consumer XSD to obtain a list of the semantically annotated [10][11] XSD elements that the consumer must receive, grouped by group IDs (subsection III-C) (if a consumer XSD element is mandatory and does not have semantic annotations, the tool stops and reports that cannot generate the translator);

3) parses the provider XSD to obtain a list of the semantically annotated XSD elements that the provider sends, also grouped by group IDs;

4) for each consumer group (of XSD elements), checks if an appropriate provider group (of XSD elements) exits (if not, the tool stops and reports that cannot 
generate the translator) - a provider group is appropriate if: (1) for each consumer XSD element, exists a semantically compatible XSD element or complement data (subsection III-D) in the provider group or (2) for each consumer XSD element that has data values that are mapped into ontology concepts [12], the provider semantically compatible XSD element does not have data values mapped into individuals that are not mapped in the consumer XSD element;

5 ) if the tool does not stop, it generates an XSLT capable of creating the consumer XML structure with the values that extract from the consumer XML message.

The developed tool uses the Pellet semantic reasoner [38]. Pellet is an OWL-DL (Web Ontology Language - Description Logic) reasoner. Pellet includes reasoning capabilities about, not only, but also the following elements [39]: (1) subClassOf - Defines a hierarchy between concepts. It means that each concept is related in the form of parent/child. As a result, a child concept with a subclassof property, will receive all characteristics (e.g., relations) of its parent; (2) subPropertyOf - Refers hierarchy of concept properties (e.g., relations between concepts). In-depth, if a property is a child of another property, it means that its elements are also related by the parent relation; (3) equivalentClass - States the equivalency of two or more classes (i.e., synonyms). Equivalent classes share same features (e.g., instances); (4) sameAs - Used to express that two individuals (with different names/URIs) are the same; and (5) equivalentProperty - Expresses that two properties are equivalent.

The tool prototype was developed as a web client-server system, using PHP for web server side, Java, OWL-API [40], and Pellet for Semantic Engine at the server, and HTML/JS for Front-End UI side. The tool also has a command line interface. The tool is available online at http://gres.uninova.pt/tag/.

\section{EXPERIMENTAL EVALUATION}

To evaluate our contributions in the domain of the Arrowhead Framework (AF), we generated translators for real world interaction scenarios of the IoT domain. Heterogeneous systems (the providers) that provide data - about temperature modules - to other heterogeneous systems (the consumers), were considered. These providers could be for instance in a city, providing the temperature of specific locations. The providers could provide data about one or more temperature sensors and, for each sensor the system could provide the temperature value and units and the sensor location (latitude and longitude). To be discovered, the providers must be registered in the AF (Fig. 1 - Registration (1)). Systems that consume data about temperature modules (the consumers), must search for providers in the AF (Fig. 1 - Discovery (1)).

Six providers and five consumers, that send/receive heterogeneous XML messages, were considered in the validation process, making a total of $30(6 \times 5)$ interaction scenarios. The used provider XML messages are equal to those sent by the following industrial/commercial systems that could be used in a smart city, which is an Arrowhead Framework application domain:
1) the ControlByWeb $\mathrm{X}-\mathrm{DAQ}-2 \mathrm{R} 1-4 \mathrm{~T}-5 / \mathrm{I} / \mathrm{E}$ temperature module [41];

2) the ControlByWeb $X 300$ temperature module [42] in Thermostat Mode;

3) the ServersCheck SensorGateway [43];

4) the Smartbedded Meteohub [44];

5) the Weather Underground weather station [45];

6) the X300 temperature module [42] in Temperature Monitor Mode.

Given that the six providers XSDs were not available/found, they were automatically generated, using the available XMLs as inputs in the XSD/XML Schema Generator (FreeFormatter.com) [46], and then a few minor changes were made to the XSDs, for tuning them. The generated providers XSDs are available online at http://gres.uninova.pt/tag/validation/XSD -Providers-withoutAnnot/.

The consumers XSDs were created almost as if they were consumers of the providers listed above. They are available online at http://gres.uninova.pt/tag/validation/XSD-Consumers -withoutAnnot/. These XSDs were created, during this work, with the following assumptions about the consumers: must receive an XML message; must receive data about one or more temperature sensors; for each temperature sensor must receive the current temperature value; for each temperature sensor can receive the temperature units; for each temperature sensor can receive its location (latitude and longitude); for each temperature sensor can receive its location units. The five consumers XSDs are available online at http://gres.uninova.pt/tag/validation/XSD-Providers -withoutAnnot/.

Given that the previous providers and consumers XSDs do not include semantic annotations, required to support the semantic compatibility verification and the automatic creation of XML translators, these XSDs were extended with annotations: (1) path based semantic annotations and (2) semantic annotation of data. Additionally, group identifiers (IDs) (proposed in this paper) were introduced in the semantic annotations of the XSDs to avoid ambiguities. Finally, complement data values with semantic annotations (also proposed in this paper) were added to provide fixed data about the providers that the consumers must receive. Semantic annotation of data was used to associate temperature units tags with the ontology individuals. Group IDs were used for instance to associate temperature sensors with their location. Complement property value annotations were used for instance to introduce the location latitude and longitude values into the XSDs. These annotations enable the compatibility verification and the automatic creation of XML translators. The resulting providers and consumers XSDs are online at http://gres.uninova.pt/tag/validation/XSD -Providers/ and http://gres.uninova.pt/tag/validation/XSD -Consumers/.

An overview of the data provided by the providers and how they are distributed by their XML messages and associated XSDs, is presented in table I. The row "No. of temp. sensors" presents the number of temperature values that each provider sends and between parenthesis specifies, when applicable, if the values are indoor or outdoor temperatures. The provider 
" 5 " is the only one that sends the temperature value in Fahrenheit (F) and Celsius (C). The row "Temperature units" specifies if the units are sent in the XML messages (providers " 1 ", " 2 ", and "6" send "F" or " $C$ ") or not. When not sent in the XML, they are introduced in the XSDs as semantic annotations, which means that the sent temperature values have always the same units. The row "Sensor location" specifies if the location is sent in the XML messages or not. Only provider " 5 " sends location, which means that the other sensors are always in the same place. For the other providers the location was introduced in the XSDs using the complement data annotations proposed in this paper. Finally, the last row specifies that the location units is always fixed in the XSDs.

TABLE I

THE DATA PROVIDED B Y THE PROVIDERS AND HOW THEY ARE DISTRIBUTED B Y THEIR XML MESSAGES AND ASSOCIATED XSDS

\begin{tabular}{|c|c|c|c|c|}
\hline Provider & $\begin{array}{c}\text { No. of temp. } \\
\text { sensors }\end{array}$ & $\begin{array}{c}\text { Temperature units } \\
\text { Sensor } \\
\text { location }\end{array}$ & $\begin{array}{c}\text { Location } \\
\text { units }\end{array}$ \\
\hline 1 & 2 & Variable (XML) - F or C & Fixed (XSD) & Fixed (XSD) \\
\hline 2 & 2 (1in, 1 out) & Variable (XML) - F or C & Fixed (XSD) & Fixed (XSD) \\
\hline 3 & $2(1$ out) & Fixed (XSD) - C & Fixed (XSD) & Fixed (XSD) \\
\hline 4 & $3(1$ in, 1 out) & Fixed (XSD) - C & Fixed (XSD) & Fixed (XSD) \\
\hline 5 & 1 (F and C) & Fixed (XSD) - F and C & Variable (XML) & Fixed (XSD) \\
\hline 6 & 7 & Variable (XML) - F or C & Fixed (XSD) & Fixed (XSD) \\
\hline
\end{tabular}

Each pair XSDs (with the proposed annotations), used as input in the developed tool prototype, supported the verification of the systems (one provider and one consumer) interoperability and, when possible, the automatic generation of the required translator. The annotated XSDs supported the automatic generation of translators in $80 \%$ of the interacting scenarios (24 of 30). It is not possible to generate translators to support the communication from: provider 1 to consumer 2 (P1 to $\mathrm{C} 2$ ); $\mathrm{P} 5$ to $\mathrm{C} 2$; and $\mathrm{P} 6$ to $\mathrm{C} 2$, because consumer $\mathrm{C} 2$ must receive an outdoor temperature but the three providers $(\mathrm{P} 2$, $\mathrm{P} 5$, and P6) send temperatures without specifying if they are exterior or not. In the other three interaction scenarios (P1 to $\mathrm{C} 4, \mathrm{P} 2$ to $\mathrm{C} 4$, and $\mathrm{P} 6$ to $\mathrm{C} 4$ ) the consumer $\mathrm{C} 4$ can only receive temperature values in Celsius, but the providers (P1, P2, and P6) can send also in Fahrenheit. The 24 translators are online at http://gres.uninova.pt/tag/validation/XSLT-Ready/. Without the annotations proposed in this paper it would be only possible to generate translators in 14 of 30 (47\% success rate). It is not possible in scenarios where the consumer receives sensor locations and the provider sends data about two or more temperature sensors (groups were used to associate sensors to their locations in the XSDs). These results are summarized in Table II.

\section{CONCLUSiOnS}

This paper contributions are results of the work developed in the context of the Arrowhead EU project. During the work the following research questions arise: (1) Is it possible to verify, before consumer requests/subscriptions if it can understand provider messages? and (2) Is it possible to automatically generate data translators that enable the interoperability between heterogeneous providers/consumers?

In order automatically verify systems interoperability and generate the required translators, it was required to have proper
TABLE II

TRANSLATORS' AUTOMATIC GENERATION SUCCESS RATES OF THE CONSIDERED INTERACTION SCENARIOS

\begin{tabular}{|c|c|c|c|c|c|c|c|c|c|c|}
\hline \multicolumn{6}{|c|}{ Without groups \& property values } & \multicolumn{5}{|c|}{ With groups \& property values } \\
\hline & C1 & C2 & C3 & C4 & C5 & C1 & C2 & C3 & C4 & C5 \\
\hline P1 & OK & & & & & OK & & OK & & OK \\
\hline P2 & OK & OK & & & & OK & OK & OK & & OK \\
\hline P3 & OK & OK & & OK & & OK & OK & OK & OK & OK \\
\hline P4 & OK & OK & & OK & & OK & OK & OK & OK & OK \\
\hline P5 & OK & & OK & OK & OK & OK & & OK & OK & OK \\
\hline \multirow[t]{2}{*}{ P6 } & OK & & & & & OK & & OK & & OK \\
\hline & \multicolumn{5}{|c|}{$47 \%$ SUCCESS RATE } & \multicolumn{5}{|c|}{$80 \%$ SUCESS RATE } \\
\hline
\end{tabular}

meta-data about the data sent/received by the providers/consumers and use it as input in a tool. Given that, another research question arises: How to describe the message format, the message elements, and the elements meaning? The answer to this question was: Semantically annotated XML-Schema!

It was possible to conclude, by using the existing annotation methods for XML-Schemas to support interoperability, that these methods should be extended to increase the success rates. The introduction of group identifiers, was proposed in this paper, to solve ambiguities; whereas the introduction of complement data values into XML-Schemas, also proposed in this paper, enable the interaction of providers that send messages with less data than the one required by the consumers (this data is then added by the translators).

A tool prototype has been developed during this work in order to provide a proof of concept of the present paper contributions. With such tool we were able to automate the interoperability between XML based devices. By annotating their XSDs, and mapping them against a domain ontology, we were able to generate their translators. Data translators such as units converters (for instance to convert Celsius to Fahrenheit) are planned to be implemented in the future.

The experimental evaluation shows what was expected, that: (1) it is possible to automatically verify, before systems interaction, if they can interact; (2) it is possible to automatically generate data translators that support systems interoperability, with our tool prototype. In the presented 30 interaction scenarios we achieve, by using XML-Schemas with semantic annotations (not extended), an automation success rate of $47 \%$. Such rate achieved $80 \%$ when using the extended semantic annotations.

The approach presented in this paper was framed by the Arrowhead project and targeted the generation of translators for XML messages in the IoT domain. Our experiments clearly show the applicability of the approach in the given setting. We see two interesting areas of future work: (1) the application and evaluation of the proposed approach in other domains with potentially more complex schemas and (2) the semi-automatic generation of annotations based on matching XML-Schemas and ontologies.

\section{REFERENCES}

[1] V. Vyatkin, "IEC 61499 as Enabler of Distributed and Intelligent Automation: State-of-the-Art Review," IEEE Trans. Ind. Informat., vol. 7, no. 4, pp. 768-781, Nov 2011.

[2] V. Jirkovský, M. Obitko, and V. Mařík, "Understanding data heterogeneity in the context of cyber-physical systems integration," IEEE Trans. Ind. Informat., vol. 13, no. 2, pp. 660-667, April 2017. 
[3] L. D. Xu, W. He, and S. Li, "Internet of things in industries: A survey," IEEE Trans. Ind. Informat., vol. 10, no. 4, pp. 2233-2243, Nov 2014.

[4] Z. D. Zhou, R. Valerdi, S. M. Zhou, and L. Wang, "Guest Editorial: Special Section on IoT," IEEE Trans. Ind. Informat., vol. 10, no. 2, pp. 1413-1416, May 2014

[5] G. Xiao, J. Guo, L. D. Xu, and Z. Gong, "User Interoperability With Heterogeneous IoT Devices Through Transformation," IEEE Trans. Ind. Informat., vol. 10, no. 2, pp. 1486-1496, May 2014.

[6] IERC, "IoT Semantic Interoperability: Research Challenges, Best Practices, Recommendations and Next Steps," European Research Cluster On The Internet Of Things, Brussels: European Commission, Tech. Rep., Mar. 2015. [Online]. Available: http://www.internet-of-thingsresearch.eu/pdf/IERC_Position_Paper_IoT_Semantic_Interoperability_ Final.pdf

[7] F. Blomstedt, L. L. Ferreira, M. Klisics, C. Chrysoulas, I. M. de Soria, B. Morin, A. Zabasta, J. Eliasson, M. Johansson, and P. Varga, "The arrowhead approach for soa application development and documentation," in IECON 2014 - 40th Annual Conference of the IEEE Industrial Electronics Society, Oct 2014, pp. 2631-2637.

[8] (2017) Arrowhead I Ahead of the future. [Online]. Available: http://www.arrowhead.eu/

[9] P. M. N. Maló, "Hub-and-spoke Interoperability: an out of the skies approach for large-scale data interoperability," Ph.D. dissertation, FCTUNL, 2013.

[10] J. Köpke and J. Eder, "Semantic annotation of xml-schema for document transformations," in Proc. of OTM'10 Workshops, ser. LNCS, vol. 6428. Springer, 2010, pp. 219-228.

[11] J. Köpke, "Annotation Paths for Matching XML-Schemas," forthcoming. Available: http://isys.uni-klu.ac.at/PDF/prep/AnnotationPathMatching.p df

[12] F. Moutinho, L. Paiva, P. Maló, and L. Gomes, "Semantic annotation of data in schemas to support data translations," in IECON 2016 - 42nd Annual Conference on IEEE Industrial Electronics Society, Oct. 2016.

[13] W. OWL Working Group, OWL 2 Web Ontology Language: Document Overview. W3C Recommendation, 27 October 2009, available at http://www.w3.org/TR/owl2-overview/.

[14] J. Kahan and M.-R. Koivunen, "Annotea: an open rdf infrastructure for shared web annotations," in Proc. of the 10th international conference on World Wide Web, ser. WWW '01. New York, NY, USA: ACM, 2001, pp. 623-632.

[15] S. Handschuh and S. Staab, "Authoring and annotation of web pages in CREAM," in Proc. of the 11th international conference on World Wide Web, ser. WWW '02. New York, NY, USA: ACM, 2002, pp. 462-473.

[16] J. Heflin and J. Hendler, "A Portrait of the Semantic Web in Action," IEEE Intelligent Systems, vol. 16, no. 2, pp. 54-59, Mar. 2001.

[17] M. Sporny, M. Birbeck, I. Herman, and B. Adida, "RDFa 1.1 primer - third edition," W3C, W3C Note, Mar. 2015, http://www.w3.org/TR/2015/NOTE-rdfa-primer-20150317/.

[18] P. Ciccarese, B. Young, and R. Sanderson, "Web Annotation Data Model," W3C, Candidate Recommendation, Nov. 2016, https://www.w3.org/TR/2016/CR-annotation-model-20161122/.

[19] J. Kopecký, T. Vitvar, C. Bournez, and J. Farrell, "SAWSDL: Semantic Annotations for WSDL and XML Schema," IEEE Internet Computing, vol. 11, no. 6, pp. 60-67, Nov 2007.

[20] L. Popa, Y. Velegrakis, M. A. Hernández, R. J. Miller, and R. Fagin, "Translating web data," in Proc. of the 28th international conference on Very Large Data Bases, ser. VLDB '02. VLDB Endowment, 2002, pp. 598-609.

[21] G. Mecca and P. Papotti, "Schema mapping and data exchange tools: Time for the golden age." it - Information Technology, vol. 54, no. 3, pp. 105-113, 2012.

[22] R. Fagin, P. G. Kolaitis, R. J. Miller, and L. Popa, "Data exchange: semantics and query answering," Theoretical Computer Science, vol. 336, no. 1, pp. $89-124,2005$.

[23] J. Köpke and J. Eder, "Logical invalidations of semantic annotations," in Advanced Information Systems Engineering: 24th International Conference, CAiSE 2012, Gdansk, Poland, June 25-29, 2012. Proceedings, ser. LNCS, vol. 7328. Berlin, Heidelberg: Springer, 2012, pp. 144-159.

[24] J. Köpke, J. Eder, and D. Joham, "Towards path-based semantic annotation for web service discovery," in Information Systems Engineering in Complex Environments: CAiSE Forum 2014, Thessaloniki, Greece, June 16-20, 2014, Selected Extended Papers, ser. LNBIP, vol. 204. Cham: Springer International Publishing, 2015, pp. 133-147.

[25] M. Szymczak and J. Köpke, "Matching methods for semantic annotation-based xml document transformations," in New Developments in Fuzzy Sets, Intuitionistic Fuzzy Sets, Generalized Nets and Related
Topics, ser. Applications, vol. 2. Warsaw, Poland: SRI-PAS, September 2012, pp. 297-308

[26] H.-H. Do and E. Rahm, "COMA: A system for flexible combination of schema matching approaches," in Proc. of the 28th international conference on Very Large Data Bases, ser. VLDB '02. VLDB Endowment, 2002, pp. 610-621.

[27] D. Aumueller, H.-H. Do, S. Massmann, and E. Rahm, "Schema and ontology matching with COMA++," in Proc. of the 2005 ACM SIGMOD Int'l. Conf. on Management of Data, 2005, pp. 906-908.

[28] J. Madhavan, P. A. Bernstein, and E. Rahm, "Generic Schema Matching with Cupid," in Proc. of the 27th International Conference on Very Large Data Bases, ser. VLDB '01. San Francisco, CA, USA: Morgan Kaufmann Publishers Inc., 2001, pp. 49-58.

[29] D. Nikovski, A. Esenther, X. Ye, M. Shiba, and S. Takayama, "Matcher composition methods for automatic schema matching," in Enterprise Information Systems: 14th International Conference, ICEIS 2012, Wroclaw, Poland, June 28 - July 1, 2012, Revised Selected Papers, ser LNBIP, vol. 204. Berlin, Heidelberg: Springer, 2013, pp. 108-123.

[30] J. Kim, Y. Peng, N. Ivezic, and J. Shin, "An optimization approach for semantic-based XML Schema matching," International Journal of Trade, Economics and Finance, vol. 2, no. 1, p. 78, 2011.

[31] H. Q. Thang and V. S. Nam, "XML Schema Automatic Matching Solution," International Journal of Electrical, Computer, and Systems Engineering 4:1 2010, vol. 4, no. 1, pp. 68-74, 2010.

[32] M. Missikoff, F. Schiappelli, and F. Taglino, "A controlled language for semantic annotation and interoperability in e-business applications," in Proc. of Workshop on Semantic Integration, 2003, pp. 1-6.

[33] G. Callegari, M. Missikoff, O. M, and F. Taglino, "Semantic Annotation language and tool for Information and Business Processes - Appendix F: User Manual, ATHENA Deliverable D.A3.3,' LEKS (Laboratory for Enterprise Knoweldge and Systems), Tech. Rep., 2006.

[34] T. Pankowski and R. Udalowski, "Generating schema mappings based on annotations in a P2P data integration system," in Proc. of iiWAS'2010 The 12th International Conference on Information Integration and Webbased Applications and Services, 8-10 November 2010, Paris, France, G. Kotsis, D. Taniar, E. Pardede, I. Saleh, and I. Khalil, Eds. ACM, 2010, pp. 687-691.

[35] M. Vujasinovic, N. Ivezic, B. Kulvatunyou, E. Barkmeyer, M. Missikoff, F. Taglino, Z. Marjanovic, and I. Miletic, "Semantic mediation for standard-based B2B interoperability," Internet Computing, IEEE, vol. 14, no. 1 , pp. $52-63$, jan.-feb. 2010

[36] M. Krötzsch, F. Simančík, and I. Horrocks, "A description logic primer," in Perspectives on Ontology Learning. IOS Press, 2014.

[37] (2007) XSL Transformations (XSLT) Version 2.0. Accessed: April 2016. [Online]. Available: https://www.w3.org/TR/xslt20/

[38] GitHub - Complexible/pellet: Pellet is an OWL 2 reasoner in Java; open source (AGPL) and commercially licensed, commercial support available. Accessed: April 2017. [Online]. Available: https://github.com/Complexible/pellet

[39] E. Sirin, B. Parsia, B. C. Grau, A. Kalyanpur, and Y. Katz, "Pellet: A practical OWL-DL reasoner," Web Semantics: Science, Services and Agents on the World Wide Web, vol. 5, no. 2, pp. 51 - 53, 2007, software Engineering and the Semantic Web. [Online]. Available: http://www.sciencedirect.com/science/article/pii/S1570826807000169

[40] OWL API. Accessed: April 2017. [Online]. Available: http://owlapi.sourceforge.net/

[41] Temperature Module Users Manual. Accessed: March 2017. [Online]. Available: http://www.controlbyweb.com/temperature/temperature_user s_manual.pdf

[42] X300 Advanced Temperature Module Users Manual. Accessed: March 2017. [Online]. Available: http://www.controlbyweb.com/x300/x300_users_manual.pdf

[43] SERVERSCHECK User Manual. Accessed: March 2017. [Online]. Available: http://manuals.serverscheck.com/ServersCheck_Sensor_Gate way.pdf

[44] HTTP Data Logging Protocol Version 1.2 (introduced with Meteohub 4.5g). Accessed: March 2017. [Online]. Available: http://www.meteohub.de/files/HTTP-Data-Logging-Protocol-v1.2.pdf

[45] API Weather Underground. Accessed: March 2017. [Online]. Available: https://www.wunderground.com/weather/api

[46] Free Online XSD/XML Schema Generator - FreeFormatter.com. Accessed: March 2017. [Online]. Available: http://www.freeformatter.com/xsd-generator.html 


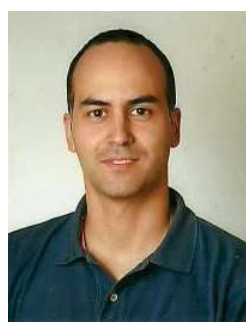

Filipe Moutinho received the Eng. degree (in 2003), the M.Sc. degree (in 2009), and the Ph.D. degree (in 2014) in Electrical and Computer Engineering from the Faculty of Science and Technology of NOVA University of Lisbon (FCT-UNL), Portugal.

$\mathrm{He}$ is an Assistant Professor with the Electrical Engineering Department, FCT-UNL, and a researcher at the Center of Technology and Systems (CTS), UNINOVA institute, FCT-UNL. He worked as a software engineer with the enterprise NewHotel Software, Lisbon. His research interests include Cyber-Physical Systems and Internet-of-Things, namely the modeling, the validation, and the automatic code generation for embedded systems and also the interoperability between them. He was researcher in several research projects, among which are the European projects with the designations Arrowhead (grant agreement nr. 332987) and Productive4.0 (grant agreement nr. 737459) and the Portuguese project with the designation Petri-Rig (ref. PTDC/EEI-AUT/2641/2012).

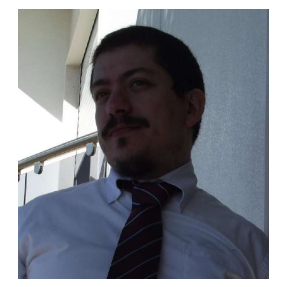

Luís Paiva received his M.Sc. in Electrical and Computer Engineering in 2015 by Faculty of Science and Technology of NOVA University of Lisbon (FCT-UNL), Portugal in 2015.

He's main current activities focus are aimed at research in IoT and Semantic areas. Currently he does research integrated in UNINOVA Research Institute from Lisbon, Portugal for 2 years. He also worked as a software engineer and developer, integrating projects in finance and insurance. Main research interests are amongst automatization of semantics, shared understanding between systems exchanging data. The projects in which he participated as a researcher and developer are EU projects, such as ProaSense (grant agreement nr. 612329), Arrowhead (grant agreement nr. 332987) and Design4Energy (grant agreement nr. 609380).

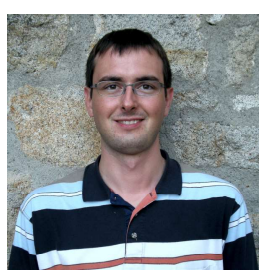

Julius Köpke is an Assistant Professor in the Department of Informatics Systems at the University of Klagenfurt (Austria). He received his $\mathrm{PhD}$ with distinction from the same university in 2012. From March 2015 to March 2016 Julius was a visiting scholar at the University of California in Santa Barbara.

His main research interests are framed by application interoperability for interorganizational cooperation. His contributions on the message and on the process level are published in conferences such as BPM or CAiSE. He served as a member of the program committee or reviewer for various prestigious conferences such as ER, BPM, CAiSE, COOPIS and for Journals such as Information Systems and Cooperative Information Systems.

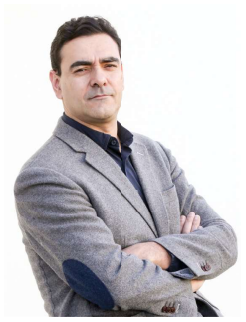

Pedro Maló is a Professor at the Electrical Engineering Department (DEE) of the Faculty of Science and Technology of NOVA University of Lisbon (FCT-UNL) and Senior Researcher at the UNINOVA Centre of Technology and Systems (CTS). He is graduated, with M.Sc. in Computer Science and a holds Ph.D. in Computer Engineering.

His core research interests are the interoperability and integrability of (complex) systems with special emphasis on Cyber-Physical Systems / Internet of Things. He has $15+$ years practice in the management, research and technical coordination/development of research and innovation projects in ICT domains especially addressing data solutions, systems' interoperability and integration technologies. He is an author of over 70 scientific publications published as book chapters, journal articles or conference papers. 